\title{
NOUVELLE
}

\section{Tryptophane et dérégulations métaboliques, un nouvel enjeu pour la santé}

Allison Agus ${ }^{1}$, Harry Sokol ${ }^{1,2}$
${ }^{1}$ Institut Micalis, INRA, AgroParisTech, Université Paris-Saclay, Domaine de Vilvert, 78352 Jouy-en-Josas, France.

${ }^{2}$ Sorbonne Université, Inserm, Centre de Recherche SaintAntoine, CRSA, AP-HP, Hôpital Saint-Antoine, Service de Gastroentérologie, F-75012 Paris, France. harry.sokol@aphp.fr
Le microbiote intestinal, une nouvelle piste pour son implication dans le syndrome métabolique

Le syndrome métabolique désigne la coexistence de plusieurs anomalies qui se traduisent généralement par une obésité, une résistance à l'insuline, une intolérance au glucose et une hyperlipidémie augmentant considérablement le risque de pathologies cardiovasculaires. Le rôle causal du microbiote intestinal dans le développement du syndrome métabolique est d'ores et dèja reconnu. En effet, au même titre que d'autres pathologies comme les maladies inflammatoires chroniques de l'intestin, le syndrome métabolique est caractérisé par un déséquilibre du microbiote intestinal appelé dysbiose, correspondant à un appauvrissement de la biodiversité et à l'accroissement de certaines espèces bactériennes aux dépens d'autres [1-3]. Notre tube digestif abrite pas moins de $10^{13}$ à $10^{14}$ micro-organismes qui constituent notre microbiote intestinal, impliqué dans un grand nombre de fonctions physiologiques essentielles pour l'organisme à la fois digestives, métaboliques, immunitaires et neurologiques [4, 5] $(\rightarrow)$.

$(\rightarrow)$ Voir la Synthèse de V. GaboriauRouthiau et N. Cerf-Bensussan, $\mathrm{m} / \mathrm{s} \mathrm{n}^{\circ} 11$, novembre 2016, page 961 La majorité des métabolites fermentaires produits par le microbiote intestinal sont utilisés par l'hôte et même si une grande partie de ces métabolites sont bénéfiques pour sa santé, certains peuvent être délétères [6]. Toute rupture d'équilibre de l'écosystème digestif peut donc engendrer des conséquences pou- vant conduire à un état pathologique [7] $(\rightarrow)$. Les différents travaux de notre équipe ont permis de montrer qu'un

$(\rightarrow)$ Voir le numéro thématique Le microbiote, $m / s n^{\circ} 11$ novembre 2016 grand nombre de métabolites participe au dialogue complexe existant entre microbiote intestinal et désordre métabolique avec, notamment, un rôle clé du métabolisme du tryptophane [8]. Cet acide aminé essentiel, précurseur de nombreux métabolites, est un acteur crucial impliqué dans diverses voies de signalisation dans l'organisme allant de l'intestin jusqu'au cerveau [9].

Le métabolisme du tryptophane:

3 voies essentielles liées au microbiote intestinal

\section{La voie AhR (aryl hydrocarbon receptor)}

Le tryptophane est transformé par certaines bactéries du microbiote en dérivés indoles qui sont capables d'activer le récepteur des hydrocarbures aromatiques $A h R$, un récepteur présent sur des cellules immunitaires et sur des cellules épithéliales intestinales. Son activation entraîne une production par les cellules immunitaires d'interleukine-22 (IL-22) qui a une action anti-inflammatoire et un rôle de protection de la muqueuse [10].

\section{La voie de la sérotonine}

Ce neurotransmetteur est produit à partir du tryptophane et a des effets sur l'ensemble de l'organisme. II intervient dans différents processus biologiques et dans de nombreuses pathologies. Plus de $80 \%$ de la sérotonine de notre organisme est fabriquée dans l'intestin par des cellules spécialisées et sous l'influence du microbiote [11].

\section{La voie IDO (indoléamine 2,3-dioxygé- nase)}

À partir du tryptophane, la voie IDO conduit notamment à la production de la kynurénine, mais aussi de nombreux autres métabolites qui sont impliqués dans des processus immunitaires, métaboliques et même neurologiques [12].

Le décryptage de l'équilibre complexe qui existe entre les différentes voies du métabolisme du tryptophane devrait donc permettre de mieux comprendre la survenue de certaines pathologies intestinales, mais aussi extra-intestinales. La connexion entre microbiote et santé laisse entrevoir le développement de nouvelles stratégies thérapeutiques, notamment dans le traitement du syndrome métabolique.

Ainsi, comment le microbiote intestinal est-il capable de moduler la réponse immunitaire de l'hôte? Et plus spécifiquement, quel est le rôle du tryptophane dans le dialogue entre l'hôte et son microbiote? Quel est le lien entre microbiote intestinal, métabolisme du tryptophane et syndrome métabolique? Pour répondre à ces questions, nous avons dressé un état des lieux des dernières avancées scientifiques sur le rôle du tryptophane et de ses métabolites dans le dialogue avec le microbiote intestinal [9]. Deux aspects principaux ont été abordés: (1) les effets des dérivés du tryptophane produits directement par les bactéries du microbiote, et (2) le contrôle indirect sur le métabolisme du 


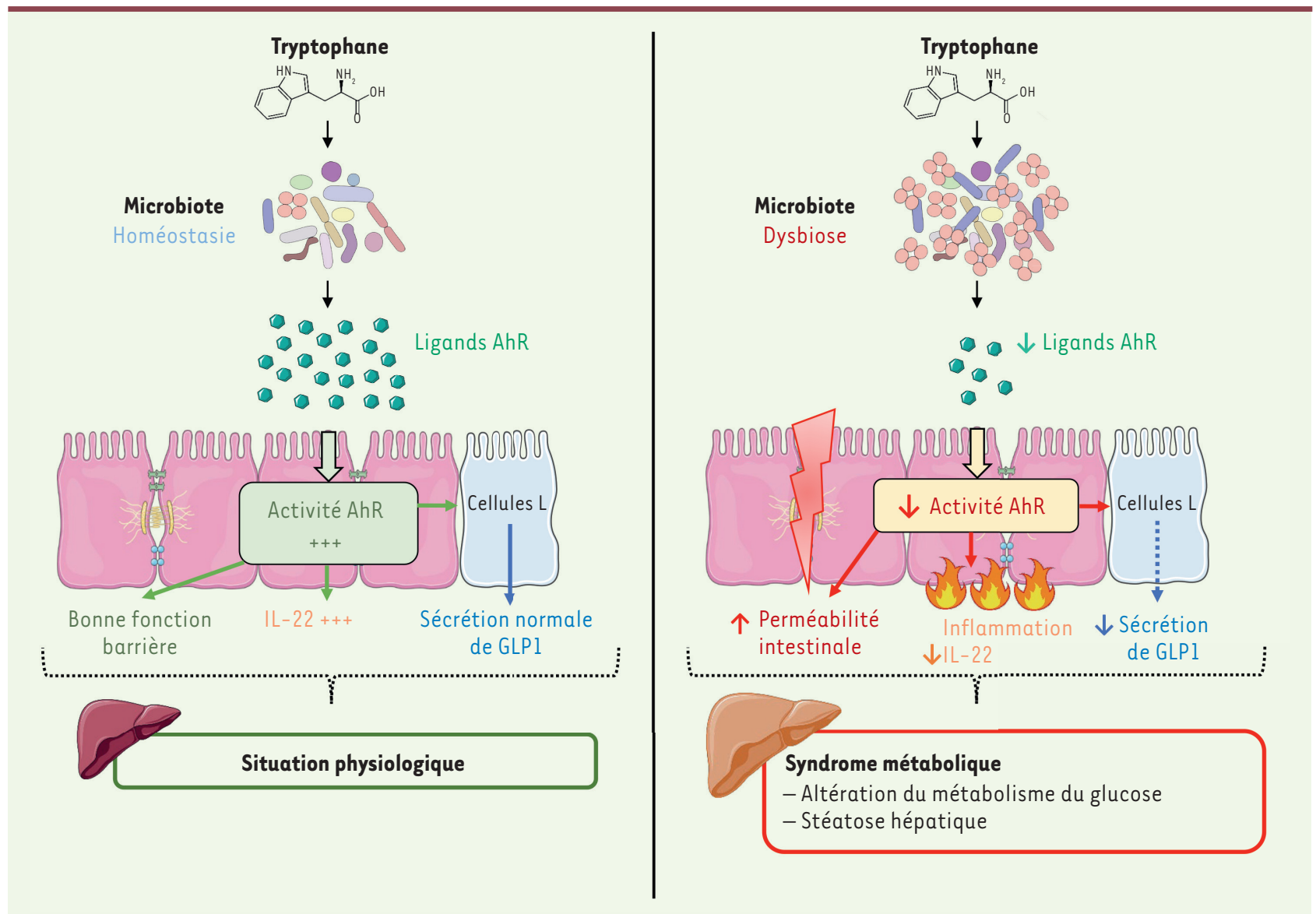

Figure 1. Modèle démontrant le lien fort entre microbiote intestinal, métabolisme du tryptophane et syndrome métabolique. Dans un contexte de syndrome métabolique, le microbiote intestinal dit «dysbiotique » est incapable, à partir du tryptophane, de produire autant d'agonistes AhR (aryl hydrocarbon receptor) que dans une situation physiologique. Ce défaut d'activation de AhR induit une diminution de production d'interleukine-22 (IL-22), une augmentation de perméabilité intestinale associée à une diminution de sécrétion de l'hormone glucoincrétine GLP-1 (glucagon-like-peptide-1). Ces multiples altérations facilitent le développement d'un syndrome métabolique qui se caractérise par un dysfonctionnement du métabolisme du glucose et une stéatose hépatique.

tryptophane de l'hôte par le microbiote intestinal. Dans notre étude, nous nous sommes tout particulièrement intéressés à la voie mettant en jeu $A h R$, le récepteur des hydrocarbures aromatiques.

\section{Altération du métabolisme du tryptophane et de la réponse} immunitaire chez les souris atteintes d'un syndrome métabolique : rôle du microbiote intestinal ?

Afin de déterminer si un lien causal existait entre microbiote intestinal, tryptophane et syndrome métabolique, nous avons tout d'abord utilisé une alimentation riche en matières grasses
(HFD, high-fat diet) afin d'induire, dans un modèle murin, un syndrome métabolique. Une diminution de l'activité de AhR associée à une plus faible production de ses ligands par le microbiote intestinal a été identifiée chez les souris HFD atteintes d'un syndrome métabolique. Chez ces mêmes souris, l'analyse transcriptomique du côlon et de l'iléon a également montré une diminution d'expression d'IL-22, de Reg3y (regenerating islet-derived protein 3 -gamma) et de Reg3 $\beta$ (regenerating islet-derived protein 3-beta), des gènes cibles de AhR $[13,14]$. La réparation tissulaire intestinale est induite en partie par I'IL-22 [15]. Cette cytokine participe aussi à la réponse immunitaire contre les infections bactériennes et fongiques via l'induction de la production des peptides antimicrobiens $\operatorname{Reg} 3 \gamma$ et $\operatorname{Reg} 3 \beta$ par les cellules épithéliales intestinales. Ces données mettent ainsi en évidence une réponse immunitaire intestinale anormale des souris souffrant d'un syndrome métabolique.

Afin de montrer l'importance du microbiote intestinal dans la mise en place du syndrome métabolique, nous avons colonisé des souris axéniques ${ }^{l}(A x)$ sauvages (WT, wild type) avec le microbiote de souris sauvages nourries avec un

\footnotetext{
${ }^{1}$ Dépourvues de germe ou germ free en anglais.
} 
régime conventionnel $\left(M_{W T-C O N V} \rightarrow A x\right)$ ou de souris sauvages nourries avec un régime HFD ( $\left.M_{\text {WT-HFD }} \rightarrow A x\right)$. Le transfert de microbiote d'une souris WT-HFD chez une souris $A x-W T$ s'est révélé suffisant pour reproduire le phénotype observé chez les souris WT-HFD. En effet, nous avons retrouvé une activation notablement plus faible de AhR dans les fèces des souris ( MWT-HFD $_{\rightarrow} \mathrm{Ax}$ ) par rapport aux souris $\left(M_{\text {WT-CONV }} \rightarrow A x\right)$. L'ensemble de ces résultats montre ainsi un défaut du métabolisme du tryptophane par le microbiote intestinal des souris HFD qui induit une diminution de l'activité $A h R$ et de la production d'IL-22.

Diminution de l'activité AhR et des métabolites du tryptophane chez les patients atteints d'un syndrome métabolique

Afin de déterminer la pertinence de nos résultats chez l'homme, nous avons analysé l'activité $A h R$ de fèces issues de volontaires sains et de patients atteints du syndrome métabolique. Une diminution de l'activité AhR associée à une réduction des concentrations en agonistes $A h R$, tels que l'indole ou encore l'acide indole-3-acétique, a été observée chez des patients atteints du syndrome métabolique. Ces résultats montrent que le métabolisme du tryptophane par le microbiote intestinal des patients atteints de syndrome métabolique est altéré et conduit à un défaut d'activation de AhR.

\section{Les agonistes AhR comme nouvelle perspective thérapeutique}

Afin de déterminer si les altérations de production d'agonistes AhR par le microbiote jouaient un rôle dans la sévérité du syndrome métabolique, nous avons corrigé ce défaut de production en supplémentant l'alimentation des souris avec des agonistes AhR ou des souches probiotiques de nouvelle génération (de type Lactobacilles) produisant naturellement des ligands de AhR. Cette stratégie a permis d'améliorer très nettement le syndrome métabolique. Une amélioration de la tolérance au glucose et de la stéatose hépatique ont été observées, s'expliquant par une meilleure fonction de la barrière intestinale et une plus grande sécrétion de I'hormone glucoincrétine GLP-1 (glucagon-like-peptide-1)2.

De manière plus générale, notre étude a permis de mettre en évidence un lien fort entre microbiote intestinal et syndrome métabolique (Figure 1). En effet, la capacité réduite du microbiote à produire des ligands de AhR conduit à un défaut de barrière intestinale et à une diminution de la production de GLP-1, facilitant ainsi le développement d'un syndrome métabolique. Il est important de noter que ces résultats sont pertinents chez l'homme où la diminution de production d'agonistes $A h R$ est aussi observée chez les patients atteints du syndrome métabolique. En plus de fournir des preuves essentielles sur le rôle du microbiote intestinal dans le maintien de l'homéostasie métabolique chez I'hôte, ces travaux démontrent l'efficacité pharmacologique de molécules ou de bactéries probiotiques sélectionnées sur leur capacité fonctionnelle à corriger le déficit en ligands de AhR. En conclusion, cette étude ouvre la voie à de nou-

${ }^{2}$ Une hormone qui stimule la sécrétion d'insuline de manière glucose-dépendante. veaux traitements préventifs ou curatifs du syndrome métabolique. $\diamond$

Tryptophan and metabolic syndrome, a new challenge for health

\section{LIENS D'INTÉRÊT}

Les auteurs déclarent n'avoir aucun lien d'intérêt concernant les données publiées dans cet article.

\section{RÉFÉRENCES}

1. Everard A, Cani PD. Diabetes, obesity and gut microbiota. Best Pract Res Clin Gastroenterol 2013 ; $27: 73-83$.

2. Ley RE, Bäckhed F, Turnbaugh $P$ et al. Obesity alters gut microbial ecology. Proc Natl Acad Sci USA 2005 ; 102 : 11070-5.

3. Ley RE, Turnbaugh PJ, Klein S, et al. Microbial ecology: human gut microbes associated with obesity. Nature 2006 ; 444 : 1022-3.

4. Gaboriau-Routhiau V, Cerf-Bensussan N. Microbiote intestinal et développement du système immunitaire. Med Sci (Paris) 2016 ; 32 : 961-7.

5. Le microbiote : cet inconnu qui réside en nous (numéro thématique). Med Sci(Paris) 2016 ; 32 : 9191037.

6. Blacher $\varepsilon$, Levy M, Tatirovsky $\varepsilon$, et al. Microbiomemodulated metabolites at the interface of host immunity. J Immunol 2017 ; 198 : 572-80.

7. Schirmer M, Smeekens SP, Vlamakis $\mathrm{H}$ et al. Linking the human gut microbiome to inflammatory cytokine production capacity. Cell 2016; $67: 1125-36$.

8. Mallmann NH, Lima ES, Lalwani P. Dysregulation of tryptophan catabolism in metabolic syndrome. Metab Syndr Relat Disord 2018 ; 16 : 135-42.

9. Agus A, Planchais J, Sokol H. Gut microbiota regulation of tryptophan metabolism in health and disease. Cell Host Microbe 2018 ; 23 : 716-24.

10. Lamas B, Natividad JM, Sokol H. Aryl hydrocarbon receptor and intestinal immunity. Mucosal Immunol 2018 ; 11 : 1024-38.

11. Gershon MD. 5-Hydroxytryptamine (serotonin) in the gastrointestinal tract. Curr Opin Endocrinol Diabetes Obes $2013 ; 20: 14-21$.

12. Wu H, Gong J, Liu Y. Indoleamine 2, 3-dioxygenase regulation of immune response. Mol Med Rep 2018 ; $17: 4867-73$.

13. Sonnenberg GF, Fouser LA, Artis D. Border patrol: regulation of immunity, inflammation and tissue homeostasis at barrier surfaces by IL-22. Nat Immunol 2011 ; 12 : 383-90.

14. Stelter C, Käppeli R, König C, et al. Salmonellainduced mucosal lectin RegllI $\beta$ kills competing gut microbiota. PLoS One 2011; 6 : e20749.

15. Pickert $G$ et al., STAT3 links IL-22 signaling in intestinal epithelial cells to mucosal wound healing. J Exp Med $2009 ; 206: 1465-72$

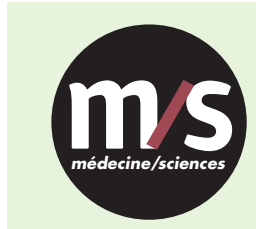

Tarifs d'abonnement m/s - 2019

Abonnez-vous

à médecine/sciences
$>$ Grâce à $m / s$, vivez en direct les progrès des sciences biologiques et médicales

Bulletin d'abonnement page 278 dans ce numéro de $\mathrm{m} / \mathrm{s}$

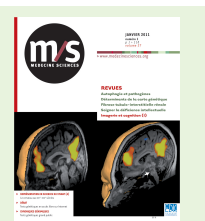

